\title{
Kiefer ordering of simplex designs for first- and second-degree mixture models
}

\author{
Norman R. Draper a, ${ }^{*}$, Friedrich Pukelsheim b,* \\ a Department of Statistics, University of Wisconsin-Madison, Madison WI 53706-1685, USA \\ b Institut für Mathematik, Universität Augsburg, D-86135 Augsburg, Germany
}

\begin{abstract}
For mixture models on the simplex, we discuss the improvement of a given design in terms of increasing symmetry as well as obtaining a larger moment matrix under the Loewner ordering. The two criteria together constitute the Kiefer design ordering. The latter can be discussed either in the usual Scheffé model algebra, or in Kronecker product algebra which better reflects the symmetries of the simplex experimental region. For the first-degree mixture model, we show that the vertex points design is the unique optimal design under the Kiefer ordering. For the second-degree mixture models with two or three ingredients, complete class results relative to the Kiefer ordering are derived. A brief overview of related literature completes the paper.
\end{abstract}

1991 Mathematics Subject Classifications: Primary 62K99, 62J05; secondary 15A69, 15A45.

Keywords: Complete class results for the Kiefer design ordering; Kronecker product; Loewner matrix ordering; Matrix majorization; Moment identities; Permutation invariant designs; Scheffé canonical polynomials; Weighted centroid designs.

\section{Introduction}

Many practical problems are associated with the investigation of mixture ingredients $t_{1}, t_{2}, \ldots, t_{m}$ of $m$ factors, with $t_{i} \geq 0$ and further restricted by $\sum t_{i}=1$. The definitive text Cornell (1990) lists numerous examples and provides a thorough discussion of both theory and practice. Early seminal work was done by Scheffé $(1958,1963)$ in which he suggested (1958, page 347) and analyzed canonical model forms of degrees one, two and three for the expected response. We shall refer to these as the S-models, or S-polynomials.

\footnotetext{
* E-mail addresses: Draper@Stat.Wisc.Edu, Pukelsheim@Uni-Augsburg.De.
} 
In this paper, we use an alternative representation of mixture models which appears to have certain advantages; see Draper and Pukelsheim (1998b). Our versions are based on the Kronecker algebra of vectors and matrices, and give rise to model functions that are homogeneous in $t_{1}, \ldots, t_{m}$. We shall refer to the corresponding expressions as the $\mathrm{K}$-models, or K-polynomials. An outline of the present paper is as follows.

In Section 2 we review the S- and K-models. Section 3 discusses exchangeability in first degree models. Section 4 shows that the unique Kiefer optimal design for the first degree model is the vertex points design.

Exchangeability in second degree models is discussed in Section 5. Section 6 treats the two factor case which involves fourth order moments $\mu_{4}, \mu_{31}$ and $\mu_{22}$. The three factor case in Section 7 requires the additional moment $\mu_{211}$. Cases for four or more factors are considerably more complicated and are discussed in Draper, Heiligers and Pukelsheim (1998).

Section 8 consists of a concise review of the relevant literature.

\section{Mixture models of type $S$ and $K$}

We consider multifactor experiments, for $m$ deterministic ingredients that are assumed to influence the response only through the percentages or proportions in which they are blended together. For $i=1, \ldots, m$, let $t_{i} \in[0,1]$ be the proportion of ingredient $i$ in the mixture. As usual, we assemble the individual components to form the vector of experimental conditions,

$$
t=\left(t_{1}, \ldots, t_{m}\right)^{\prime}
$$

The domain of variation for the column vector $t$, the experimental domain $\mathcal{T}$, then is the standard probability simplex in the space $\mathbb{R}^{m}$. Let $1_{m}=(1, \ldots, 1)^{\prime} \in \mathbb{R}^{m}$ be the unity vector, whence $1_{m}^{\prime} t=t_{1}+\cdots+t_{m}$ is the sum of the components of the vector $t$. Thus the experimental domain $\mathcal{T}$ can be represented as

$$
\mathcal{T}=\left\{t \in[0,1]^{m}: 1_{m}^{\prime} t=1\right\}
$$

Under experimental conditions $t$, the experimental response $Y_{t}$ is taken to be a scalar random variable. Replications under identical experimental conditions, or responses from distinct experimental conditions are assumed to be of equal (unknown) variance $\sigma^{2}$, and uncorrelated. Mixture models were introduced by Scheffé in his seminal (1958) paper, with 
a special emphasis on the consequences of the simplex restriction $1_{m}^{\prime} t=1$. The simplex restriction entails linear dependencies that we shall monitor carefully as we proceed.

In this paper, we deal with first- and second-degree polynomials in $t$ to model the expected response, $\mathrm{E}\left[Y_{t}\right]$. The first-degree model is

$$
\mathrm{E}\left[Y_{t}\right]=\sum_{i=1}^{m} t_{i} \theta_{i}=t^{\prime} \theta
$$

This model does not include an overall effect $\theta_{0}$. The reason is that $\theta_{0}$ would be confounded with the linear effects, through $\theta_{0}=\left(t_{1}+\cdots+t_{m}\right) \cdot \theta_{0}=\sum_{i=1}^{m} t_{i} \theta_{0}$.

For the second-degree model, Scheffé (1958) overcomes the parameter redundancy by deliberately choosing to include only the linear terms and the crossproduct terms,

$$
\mathrm{E}\left[Y_{t}\right]=\sum_{i=1}^{m} t_{i} \beta_{i}+\sum_{i=1}^{m} \sum_{j>i}^{m} t_{i} t_{j} \beta_{i j} .
$$

Draper and Pukelsheim (1998b) refer to this model as the second-degree S-model, and to its regression function as the second-degree S-polynomial.

As an alternative, Draper and Pukelsheim (1998b) propose a representation involving the Kronecker square $t \otimes t$, the $m^{2} \times 1$ vector consisting of the squares and cross products of the components of $t$ in lexicographic order of the subscripts. This is referred to as the $\mathrm{K}$-model, with a K-polynomial as the regression function,

$$
\mathrm{E}\left[Y_{t}\right]=\sum_{i=1}^{m} \sum_{j=i}^{m} t_{i} t_{j} \theta_{i j}=(t \otimes t)^{\prime} \theta .
$$

The parameters $\beta_{i}$ and $\beta_{i j}$ of the S-model are in a one-to-one relation with the parameters of the K-model parameters $\theta_{i j}$, if $\theta_{i j}=\theta_{j i}$ is assumed. See Draper and Pukelsheim (1998b) for details.

In the present paper we derive results for the Kiefer ordering of experimental designs. We emphasize elsewhere (Draper and Pukelsheim 1998a, Section 6) that the Kiefer ordering does not depend on the coordinate system that is used to represent the regression function. Hence, within the scope of the present paper, S- and K-models are equivalent. We find that the S-models deal with the model parameters in a more parsimonious manner, in contrast to the overparameterized K-models. On the other hand, the K-models more visibly reflect the symmetry structure of the problem. We shall make use of both model representations. 


\section{Exchangeability in first-degree models}

An experimental design $\tau$ on the simplex $\mathcal{T}$ is a probability measure that has finite support. Suppose the weights $w_{1}, \ldots, w_{\ell}$ are assigned to the support points $t_{1}, \ldots, t_{\ell} \in \mathcal{T}$, respectively. The experimenter is then directed to draw a proportion $w_{j}$ of all observations under experimental conditions $t_{j}$.

Given a permutation $\pi$ of the ingredients $1, \ldots, m$, we denote the corresponding permutation matrix by $R_{\pi}$,

$$
R_{\pi}=\sum_{i=1}^{m} e_{\pi(i)} e_{i}^{\prime}
$$

where $e_{i}$ designates the $i$ th Euclidean unit vector of $\mathbb{R}^{m}$, with $i$ th entry one and zeros elsewhere. The permutation matrix $R_{\pi}$ acts on the simplex $\mathcal{T}$ by usual left multiplication,

$$
R_{\pi}\left(\begin{array}{c}
t_{1} \\
\vdots \\
t_{m}
\end{array}\right)=\sum_{i=1}^{m} e_{\pi(i)} e_{i}^{\prime} t=\sum_{i=1}^{m} t_{i} e_{\pi(i)}=\sum_{i=1}^{m} t_{\pi^{-1}(i)} e_{i}=\left(\begin{array}{c}
t_{\pi^{-1}(1)} \\
\vdots \\
t_{\pi^{-1}(m)}
\end{array}\right)
$$

The action results in a permutation of the factor labels according to the inverse of $\pi$.

Let $\operatorname{Perm}(m)$ be the group of all $m \times m$ permutation matrices. A design $\tau$ is said to be permutationally invariant when

$$
\tau^{R}=\tau \quad \text { for all } R \in \operatorname{Perm}(m),
$$

where $\tau^{R}(t)=\tau\left(R^{-1} t\right)$ is the image of $\tau$ under $R$. We call a design with this invariance property an exchangeable design. It means that the design does not depend on how the labels $1, \ldots, m$ are assigned to the available ingredients. This notion, of exchangeability of a design, does not involve the regression function.

If the linear model has regression function $f(t)$, the statistical properties of a design $\tau$ are captured by its moment matrix

$$
M(\tau)=\sum_{j=1}^{\ell} w_{j} f\left(t_{j}\right) f\left(t_{j}\right)^{\prime}=\int_{\mathcal{T}} f(t) f(t)^{\prime} d \tau .
$$

In the first-degree model on the simplex, $\mathrm{E}\left[Y_{t}\right]=t^{\prime} \theta$, the regression function is the identity, $f(t)=t$. The first-degree moment matrix of the design $\tau$ thus is the $m \times m$ matrix $M(\tau)=\int_{\mathcal{T}} t t^{\prime} d \tau$. The simplex restriction has an immediate effect on moment matrices,

$$
1_{m}^{\prime} M(\tau) 1_{m}=\int_{\mathcal{T}} 1_{m}^{\prime} t t^{\prime} 1_{m} d \tau=1
$$

That is, the entries of any first-degree moment matrix sum to one, for every design on the simplex. 
The group that acts on the experimental domain $\mathcal{T}$ induces a group $\mathcal{Q}$ acting on the range of the regression function $f$. For the first-degree model on the simplex, the regression function is the identity whence the groups $\mathcal{Q}$ and $\operatorname{Perm}(m)$ coincide,

$$
f(R t) \equiv R t=Q t \equiv Q f(t) \quad \text { for all } t \in \mathcal{T} \quad \Longrightarrow \quad Q=R
$$

The group $\mathcal{Q}=\operatorname{Perm}(m)$ acts on moment matrices by congruence, $M \mapsto Q M Q^{\prime}$. A first-degree moment matrix $M$ is said to be permutationally invariant when

$$
M=R M R^{\prime} \quad \text { for all } R \in \operatorname{Perm}(m),
$$

in which case we speak of an exchangeable first-degree moment matrix.

It is evident and well known that the action of the permutation group leaves the present problem invariant. For a general discussion of invariant design problems see, for instance, Pukelsheim (1993, page 342).

Given an arbitrary design $\tau$, we obtain an exchangeable design $\bar{\tau}$ by averaging over the permutation group,

$$
\bar{\tau}=\frac{1}{m !} \sum_{R \in \operatorname{Perm}(m)} \tau^{R}
$$

If the original design $\tau$ itself is exchangeable, then no modification is necessary, $\bar{\tau}=\tau$. Otherwise the average $\bar{\tau}$ is an improvement over $\tau$, in that it exhibits more symmetry, or balancedness. In terms of matrix majorization (relative to the congruence action of the induced group $\mathcal{Q}$ ), the moment matrix of the averaged design $\bar{\tau}$ is majorized by the moment matrix of $\tau$,

$$
M(\bar{\tau}) \prec M(\tau)
$$

The terminology "is majorized by" is standard, even though for design purposes the emphasis is reversed: $M(\bar{\tau})$ is superior to $M(\tau)$ since it exhibits more symmetry. As a consequence, the design $\bar{\tau}$ yields better values than $\tau$, under a large class of optimality criteria (Pukelsheim 1993, page 349).

Symmetry and balancedness have always been a prime attribute of good experimental designs, and comprise the first step of the Kiefer design ordering. The second step concerns the usual Loewner matrix ordering. In view of the symmetrization step it suffices to search for improvement when the Loewner ordering is restricted only to exchangeable moment matrices, a much simpler task. 


\section{Kiefer optimality in the first-degree model}

The Kiefer design ordering has two steps. The first step is the majorization ordering of Section 3, to improve balancedness. The second step is an improvement relative to the usual Loewner matrix ordering within the class of exchangeable moment matrices, which we tackle now.

The first-degree moment matrix of an exchangeable design $\bar{\tau}$ is the $m \times m$ matrix

$$
M(\bar{\tau})=\left(\begin{array}{cccc}
\mu_{2} & \mu_{11} & \cdots & \mu_{11} \\
\mu_{11} & \mu_{2} & \cdots & \mu_{11} \\
\vdots & \vdots & \ddots & \vdots \\
\mu_{11} & \mu_{11} & \cdots & \mu_{2}
\end{array}\right)
$$

with identical on-diagonal entries $\mu_{2}$, the pure second moments, and identical off-diagonal entries $\mu_{11}$, the mixed second moments. The moments are the averages over the corresponding, possibly distinct individual moments of $\bar{\tau}$,

$$
\begin{gathered}
\mu_{2}=\mu_{2}(\bar{\tau})=\int_{\mathcal{T}} t_{1}^{2} d \bar{\tau}=\cdots=\int_{\mathcal{T}} t_{m}^{2} d \bar{\tau}=\int_{\mathcal{T}}\left(\frac{1}{m} \sum_{i=1}^{m} t_{i}^{2}\right) d \bar{\tau} \\
\mu_{11}=\mu_{11}(\bar{\tau})=\int_{\mathcal{T}} t_{1} t_{2} d \bar{\tau}=\cdots=\int_{\mathcal{T}} t_{m-1} t_{m} d \bar{\tau}=\int_{\mathcal{T}}\left(\frac{1}{m(m-1)} \sum_{i=1}^{m} \sum_{j \neq i} t_{i} t_{j}\right) d \bar{\tau} .
\end{gathered}
$$

The reduction by exchangeability drastically reduces the dimensionality of the problem. It leaves just two parameters, $\mu_{2}$ and $\mu_{11}$, irrespective of the number of ingredients, $m$. Furthermore, the simplex restriction

$$
1=1_{m}^{\prime} M(\bar{\tau}) 1_{m}=m \mu_{2}+m(m-1) \mu_{11}
$$

permits a final reduction to the single parameter $\mu_{2}$, say. Accordingly, the Loewner comparison of the moment matrices of two exchangeable designs amounts to comparing their second moments $\mu_{2}$, as follows.

Lemma 4.1. Let $\eta$ and $\bar{\tau}$ be two exchangeable designs on the simplex $\mathcal{T}$. Then we have

$$
M(\eta) \geq M(\bar{\tau}) \quad \Longleftrightarrow \quad \mu_{2}(\eta) \geq \mu_{2}(\bar{\tau}) .
$$

Proof. If $M(\eta) \geq M(\bar{\tau})$ then we get in particular $\mu_{2}(\eta)=e_{1}^{\prime} M(\eta) e_{1} \geq e_{1}^{\prime} M(\bar{\tau}) e_{1}=$ $\mu_{2}(\bar{\tau})$. Conversely, we assume that $\delta=\mu_{2}(\eta)-\mu_{2}(\bar{\tau}) \geq 0$. The simplex restriction yields $\mu_{11}(\eta)-\mu_{11}(\bar{\tau})=-\frac{1}{m-1} \delta$. This gives

$$
M(\eta)-M(\bar{\tau})=\frac{m}{m-1} \delta K_{m} \geq 0
$$


since the centering matrix $K_{m}=I_{m}-\frac{1}{m} 1_{m} 1_{m}^{\prime}$ is nonnegative definite. (Pukelsheim 1993, page 88).

Let $\eta_{1}$ be the vertex points design assigning uniform weights to the vertices $e_{1}, \ldots, e_{m}$ of the simplex $\mathcal{T}$,

$$
\eta_{1}\left(e_{1}\right)=\cdots=\eta_{1}\left(e_{m}\right)=\frac{1}{m}
$$

where again $e_{i}$ designates the $i$ th Euclidean unit vector of $\mathbb{R}^{m}$. The design $\eta_{1}$ is exchangeable, with moments $\mu_{2}\left(\eta_{1}\right)=1 / m$ and $\mu_{11}\left(\eta_{1}\right)=0$.

Lemma 4.2. Let $\bar{\tau}$ be an exchangeable design on the simplex $\mathcal{T}$. Then we have

$$
M\left(\eta_{1}\right) \geq M(\bar{\tau})
$$

with equality if and only if $\bar{\tau}=\eta_{1}$.

Proof. From Lemma 4.1 we have $M\left(\eta_{1}\right)-M(\bar{\tau})=\frac{m}{m-1} \delta K_{m}$, with $\delta=1 / m-\mu_{2}(\bar{\tau})$. The simplex restriction yields $\delta=(m-1) \mu_{11}(\bar{\tau}) \geq 0$. This proves $M\left(\eta_{1}\right) \geq M(\bar{\tau})$.

Equality holds if and only if $0=\mu_{11}(\bar{\tau})=\int_{\mathcal{T}} t_{i} t_{j} d \bar{\tau}$ for all $i \neq j$. Therefore the support points of $\bar{\tau}$ must be among the vertices $e_{i}$. Because of exchangeability the design $\bar{\tau}$ assigns constant weight $1 / m$ to each vertex, whence $\bar{\tau}=\eta_{1}$.

Now we view matrix majorization and Loewner ordering together, to obtain the main result on the Kiefer design ordering in first-degree models.

Theorem 4.3. Among all designs on the simplex $\mathcal{T}$, the unique Kiefer optimal design for a first-degree model is the vertex points design $\eta_{1}$, with moment matrix $M\left(\eta_{1}\right)=\frac{1}{m} I_{m}$.

Proof. Let $\tau$ be an arbitrary design on the simplex $\mathcal{T}$. Section 3 and Lemma 4.2 yield $M\left(\eta_{1}\right) \geq M(\bar{\tau}) \prec M(\tau)$. This establishes Kiefer optimality of $M\left(\eta_{1}\right)$.

Let $\tau$ be also Kiefer optimal. Then $\tau$ and $\eta_{1}$ are Kiefer equivalent, and the antisymmetry property of the Kiefer ordering (Pukelsheim 1993, page 356) entails $M(\tau)=M\left(\eta_{1}\right)$. Now Lemma 4.2 proves uniqueness, so $\tau=\eta_{1}$. 
The theorem has a companion version for rotatable first-degree moment matrices when the experimental domain is the ball, see, for instance, Pukelsheim (1993, page 389). In both cases the Kiefer optimal moment matrices are multiples of the identity matrix $I_{m}$, whence one would speak of orthogonal designs.

The Kiefer optimal design $\eta_{1}$ consists of pure "mixtures" only, and is of little practical value. The reason is the poverty of the first-degree model, not the conceptual weakness of the Kiefer ordering. Nevertheless, the discussion of the first-degree model proves instructive when we turn to higher degree models.

We append some details that are specific to the first-degree model. On the simplex, the Cauchy inequality $1=\left(1_{m}^{\prime} t\right)^{2} \leq 1_{m}^{\prime} 1_{m} \cdot t^{\prime} t$ entails $\frac{1}{m} \leq t^{\prime} t \leq 1$ for all $t \in \mathcal{T}$. Hence the pure second moment satisfies $1 / m \leq m \mu_{2} \leq 1$, and the linear relationship $m \mu_{2}+m(m-1) \mu_{11}=$ 1 implies $0 \leq m^{2} \mu_{11} \leq 1$. Thus the ranges of the second order moments are adjacent intervals,

$$
\mu_{11} \in\left[0, \frac{1}{m^{2}}\right], \quad \mu_{2} \in\left[\frac{1}{m^{2}}, \frac{1}{m}\right] .
$$

The inequality $\mu_{11} \leq \mu_{2}$ can also be deduced from $0 \leq \frac{1}{2} \int_{\mathcal{T}}\left(t_{1}-t_{2}\right)^{2} d \bar{\tau}=\mu_{2}-\mu_{11}$.

While there are plenty of exchangeable designs, just two of them suffice to generate all possible exchangeable first-degree moment matrices. To this end let $\eta_{m}$ be the overall centroid design, that is the one-point design in the overall centroid point of the simplex $\mathcal{T}$,

$$
\eta_{m}\left(\frac{1}{m} 1_{m}\right)=1
$$

Its moment matrix is $M\left(\eta_{m}\right)=\frac{1}{m^{2}} 1_{m} 1_{m}^{\prime}$. For an arbitrary exchangeable design $\bar{\tau}$ with moments $\mu_{2}$ and $\mu_{11}$ we define

$$
\alpha_{1}=m\left(\mu_{2}-\mu_{11}\right), \quad \alpha_{m}=m^{2} \mu_{11} .
$$

These two numbers satisfy $\alpha_{1} \geq 0, \alpha_{m} \geq 0$, and $\alpha_{1}+\alpha_{m}=1$. Hence the convex combination $\alpha_{1} \eta_{1}+\alpha_{m} \eta_{m}$ is a legitimate design. In fact, this design reproduces the given moments,

$$
\begin{aligned}
\mu_{2}\left(\alpha_{1} \eta_{1}+\alpha_{m} \eta_{m}\right) & =\alpha_{1} \mu_{2}\left(\eta_{1}\right)+\alpha_{m} \mu_{2}\left(\eta_{m}\right)=\mu_{2}, \\
\mu_{11}\left(\alpha_{1} \eta_{1}+\alpha_{m} \eta_{m}\right) & =\alpha_{1} \mu_{11}\left(\eta_{1}\right)+\alpha_{m} \mu_{11}\left(\eta_{m}\right)=\mu_{11} .
\end{aligned}
$$

In other words, the convex combinations of the vertex points design $\eta_{1}$ and of the overall centroid design $\eta_{m}$ exhaust all possible exchangeable first-degree moment matrices. 


\section{Exchangeability in second-degree models}

Since the experimental domain remains the simplex $\mathcal{T}$, the notion of exchangeability remains the same, too, as far as the designs themselves are concerned.

However, we now advance to the second-degree model. We choose the K-regression function $f(t)=t \otimes t$, see Draper and Pukelsheim (1998b). This choice proves convenient to determine the group $\mathcal{Q}$ that is induced on the K-regression range,

$$
f(R t) \equiv(R t \otimes R t)=Q(t \otimes t) \equiv Q f(t) \quad \text { for all } t \in \mathcal{T} \quad \Longrightarrow \quad Q=R \otimes R .
$$

Therefore the induced group consists of the Kronecker squares of all permutation matrices,

$$
\mathcal{Q}=\{R \otimes R: R \in \operatorname{Perm}(m)\}
$$

This is a proper subgroup of the permutation matrices on the space $\mathbb{R}^{m^{2}}$ where the regression function takes its values. In fact, $\mathcal{Q}$ only has order $m$ !, while $\operatorname{Perm}\left(m^{2}\right)$ has order $m^{2}$ !.

An arbitrary design $\tau$ has second-degree K-moment matrix

$$
M(\tau)=\int_{\mathcal{T}}(t \otimes t)(t \otimes t)^{\prime} d \tau
$$

As in the first-degree model, the simplex restriction has the effect that

$$
\left(1_{m} \otimes 1_{m}\right)^{\prime} M(\tau)\left(1_{m} \otimes 1_{m}\right)=\int_{\mathcal{T}}\left(1_{m}^{\prime} t\right)^{4} d \tau=1
$$

That is, the entries of any second-degree K-moment matrix sum to one.

A second-degree K-moment matrix is said to be permutationally invariant when

$$
M=(R \otimes R) M(R \otimes R)^{\prime} \quad \text { for all } R \in \operatorname{Perm}(m),
$$

in which case we speak of an exchangeable second-degree K-moment matrix. An exchangeable second-degree K-moment matrix depends on the various moments of order four. The initial cases of 2 and 3 ingredients are treated separately in Sections 6 and 7. The general case of $m \geq 4$ is dealt with in Draper, Heiligers and Pukelsheim (1998). 


\section{Two factors}

For the two-ingredient model, let $\bar{\tau}$ be an arbitrary exchangeable design on $\mathcal{T}$. Its fourth order moments are $\mu_{4}=\int t_{1}^{4} d \bar{\tau}, \mu_{31}=\int t_{1}^{3} t_{2} d \bar{\tau}, \mu_{22}=\int t_{1}^{2} t_{2}^{2} d \bar{\tau}$. The seconddegree K-moment matrix is

$$
M(\bar{\tau})=\begin{array}{llll}
t_{1} t_{1} & t_{1} t_{2} & t_{2} t_{1} & t_{2} t_{2} \\
t_{1} t_{2} \\
t_{2} t_{1} \\
t_{2} t_{2}
\end{array}\left(\begin{array}{cccc}
\mu_{4} & \mu_{31} & \mu_{31} & \mu_{22} \\
\mu_{31} & \mu_{22} & \mu_{22} & \mu_{31} \\
& & & \\
\mu_{31} & \mu_{22} & \mu_{22} & \mu_{31} \\
\mu_{22} & \mu_{31} & \mu_{31} & \mu_{4}
\end{array}\right) .
$$

Since the regression function repeats the term $t_{1} t_{2}$ as $t_{2} t_{1}$, the K-moment matrix is rank deficient. Evidently, a corresponding nullvector is $(0,1,-1,0)^{\prime}$.

The set of moments of order four determines all lower order moments. For instance, the pure third moment expands to order four by way of $\mu_{3}=\int t_{1}^{3}\left(t_{1}+t_{2}\right) d \bar{\tau}=\mu_{4}+\mu_{31}$. In this way we get the following relations:

$$
\begin{aligned}
\mu_{3} & =\mu_{4}+\mu_{31}, \\
\mu_{21} & =\quad \mu_{31}+\mu_{22} ; \\
\mu_{2}=\mu_{3}+\mu_{21} & =\mu_{4}+2 \mu_{31}+\mu_{22}, \\
\mu_{11}=\quad 2 \mu_{21} & =\quad 2 \mu_{31}+2 \mu_{22} .
\end{aligned}
$$

The simplex restriction entails

$$
\begin{aligned}
& 1=2 \mu_{4}+8 \mu_{31}+6 \mu_{22}, \\
& 1=2 \mu_{3}+6 \mu_{21}, \\
& 1=2 \mu_{2}+2 \mu_{11} .
\end{aligned}
$$

The Loewner comparison of two second-degree moment matrices can now be expressed in terms of moment conditions. Let

$$
\mu_{(2)}=\left(\mu_{2}, \mu_{11}\right)^{\prime}
$$

be the vector of moments up to order two. Because of the simplex restriction either one of the two second order moments determines the other one. Note that the first moment is constant, $\mu_{1}=1 / 2$, for all designs that are exchangeable. 
Lemma 6.1. Let $\eta$ and $\bar{\tau}$ be two exchangeable designs on the simplex $\mathcal{T}$. Then we have

$$
M(\eta) \geq M(\bar{\tau}) \quad \Longleftrightarrow \quad \mu_{(2)}(\eta)=\mu_{(2)}(\bar{\tau}), \quad \mu_{4}(\eta) \geq \mu_{4}(\bar{\tau})
$$

Proof. For the direct part we assume that $\Delta=M(\eta)-M(\bar{\tau})$ is nonnegative definite. Then $\left(1_{2} \otimes 1_{2}\right)^{\prime} \Delta\left(1_{2} \otimes 1_{2}\right)=1-1=0$ forces $\Delta\left(1_{2} \otimes 1_{2}\right)=0$, which implies $M(\eta)\left(1_{2} \otimes 1_{2}\right)=$ $M(\bar{\tau})\left(1_{2} \otimes 1_{2}\right)$. This means $\mu_{(2)}(\eta)=\mu_{(2)}(\bar{\tau})$, since

$$
M(\bar{\tau})\left(1_{2} \otimes 1_{2}\right)=\int_{\mathcal{T}}(t \otimes t)(t \otimes t)^{\prime}\left(1_{2} \otimes 1_{2}\right) d \bar{\tau}=\int_{\mathcal{T}}(t \otimes t) d \bar{\tau}=\left(\begin{array}{c}
\mu_{2}(\bar{\tau}) \\
\mu_{11}(\bar{\tau}) \\
\mu_{11}(\bar{\tau}) \\
\mu_{2}(\bar{\tau})
\end{array}\right)
$$

In addition, we have $0 \leq\left(e_{1} \otimes e_{1}\right)^{\prime} \Delta\left(e_{1} \otimes e_{1}\right)=\mu_{4}(\eta)-\mu_{4}(\bar{\tau})$.

For the converse part note that, for two ingredients, equality of second order moments implies equality of third order moments. The fourth order moment differences then are, using $\gamma=\mu_{4}(\eta)-\mu_{4}(\bar{\tau}) \geq 0$,

$$
\begin{aligned}
& \mu_{31}(\eta)-\mu_{31}(\bar{\tau})=-\gamma \\
& \mu_{22}(\eta)-\mu_{22}(\bar{\tau})=\gamma
\end{aligned}
$$

In terms of matrices this means

$$
M(\eta)-M(\bar{\tau})=\gamma\left(\begin{array}{cccc}
1 & -1 & -1 & 1 \\
-1 & 1 & 1 & -1 \\
-1 & 1 & 1 & -1 \\
1 & -1 & -1 & 1
\end{array}\right)=\gamma E \geq 0
$$

where $E=w_{12} w_{12}^{\prime}$ and $w_{12}=\left(e_{1}-e_{2}\right) \otimes\left(e_{1}-e_{2}\right)$.

Note that the condition $\mu_{4}(\eta) \geq \mu_{4}(\bar{\tau})$ could alternatively be replaced by $\mu_{31}(\eta) \leq$ $\mu_{31}(\bar{\tau})$, or by $\mu_{22}(\eta) \geq \mu_{22}(\bar{\tau})$.

A similar argument can be used to establish the corresponding result for the S-model, as follows. A second-degree S-moment matrix has the form

$$
M_{\text {S-model }}=t_{2} t_{1}\left(\begin{array}{ccc}
t_{1} & t_{2} & t_{1} t_{2} \\
t_{1} t_{2} & \mu_{11} & \mu_{21} \\
\mu_{11} & \mu_{2} & \mu_{21} \\
& & \\
\mu_{21} & \mu_{21} & \mu_{22}
\end{array}\right)
$$


The difference between the S-moment matrices of the weighted centroid design $\eta$ and of the given design $\bar{\tau}$ is

$$
\gamma\left(\begin{array}{lll}
0 & 0 & 0 \\
0 & 0 & 0 \\
0 & 0 & 1
\end{array}\right) \geq 0 .
$$

Hence Lemma 6.1 and its proof carry over with only minor adjustments.

Again the vertex points design $\eta_{1}$ and the overall centroid design $\eta_{2}$ play a special role,

$$
\eta_{1}\left(\begin{array}{l}
1 \\
0
\end{array}\right)=\eta_{1}\left(\begin{array}{l}
0 \\
1
\end{array}\right)=\frac{1}{2}, \quad \eta_{2}\left(\begin{array}{c}
\frac{1}{2} \\
\frac{1}{2}
\end{array}\right)=1 .
$$

Their moments of order four are $\mu_{4}\left(\eta_{1}\right)=\frac{1}{2}$ and $\mu_{31}\left(\eta_{1}\right)=\mu_{22}\left(\eta_{1}\right)=0$, and $\mu_{4}\left(\eta_{2}\right)=$ $\mu_{31}\left(\eta_{2}\right)=\mu_{22}\left(\eta_{2}\right)=\frac{1}{16}$. We call the designs $\eta_{1}$ and $\eta_{2}$ elementary centroid designs. They are used to generate weighted centroid designs, in the sense of the following definition.

Definition. For weights $\alpha_{1}, \alpha_{2} \geq 0$ with $\alpha_{1}+\alpha_{2}=1$, the design $\eta=\alpha_{1} \eta_{1}+\alpha_{2} \eta_{2}$ is called a weighted centroid design.

There is a simple fourth order moment check to find out whether an exchangeable design is a weighted centroid design.

Lemma 6.2. Let $\bar{\tau}$ be an exchangeable design on the simplex $\mathcal{T}$. Then we have

$$
\mu_{31}(\bar{\tau}) \geq \mu_{22}(\bar{\tau})
$$

with equality if and only if $\bar{\tau}$ is a weighted centroid design.

Proof. On the simplex $\mathcal{T}$, the function

$$
\psi\left(t_{1}, t_{2}\right)=t_{1} t_{2}\left(t_{1}-t_{2}\right)^{2}
$$

is nonnegative. This gives $\mu_{31}(\bar{\tau})-\mu_{22}(\bar{\tau})=\frac{1}{2} \int \psi\left(t_{1}, t_{2}\right) d \bar{\tau} \geq 0$.

Equality holds if and only if $\psi\left(t_{1}, t_{2}\right)=0$ for all support points $t=\left(t_{1}, t_{2}\right)^{\prime}$ of $\bar{\tau}$, which happens only for the points $(1,0)^{\prime},(0,1)^{\prime}$, or $\left(\frac{1}{2}, \frac{1}{2}\right)^{\prime}$. Because of exchangeability, $\bar{\tau}$ is a weighted centroid design. 
We are now in a position to take another look at the converse part of the proof of Lemma 6.1. There, the central quantity is the difference $\gamma$ between the pure fourth moments of $\eta$ and $\bar{\tau}$. In the special instance when $\eta$ is a weighted centroid design we may use Lemma 6.2 to obtain

$$
\mu_{31}(\bar{\tau})-\gamma=\mu_{31}(\eta)=\mu_{22}(\eta)=\mu_{22}(\bar{\tau})+\gamma
$$

whence $\gamma$ may be expressed solely in terms of moments of $\bar{\tau}$,

$$
\gamma=\frac{1}{2}\left(\mu_{31}(\bar{\tau})-\mu_{22}(\bar{\tau})\right) \geq 0
$$

Therefore, when $\eta$ is a weighted centroid design, the moment matrix difference

$$
M(\eta)-M(\bar{\tau})=\frac{1}{2}\left(\mu_{31}(\bar{\tau})-\mu_{22}(\bar{\tau})\right) c c^{\prime}
$$

is determined by quantities that solely depend on $\bar{\tau}$. This may suggest that the improving design $\eta$ itself is already determined by $\bar{\tau}$.

In order to find a weighted centroid design $\eta=\alpha_{1} \eta_{1}+\alpha_{2} \eta_{2}$ that improves upon a given exchangeable design $\bar{\tau}$, in the Loewner ordering sense of having $M(\eta) \geq M(\bar{\tau})$, Lemma 6.1 is instrumental. We determine the weights $\alpha_{1}$ and $\alpha_{2}$ by equating selected lower order moments,

$$
\begin{aligned}
& \mu_{1}(\eta) \equiv \frac{1}{2} \alpha_{1}+\frac{1}{2} \alpha_{2}=\frac{1}{2}, \\
& \mu_{11}(\eta) \equiv \quad \frac{1}{4} \alpha_{2}=\mu_{11} \text {. }
\end{aligned}
$$

The first line is trivially true since the first order moment of exchangeable designs is constant. The solutions are $\alpha_{2}=4 \mu_{11} \geq 0$, and $\alpha_{1}=1-4 \mu_{11}=2\left(\mu_{2}-\mu_{11}\right) \geq 0$. In fourth order terms we get $\alpha_{2}=8\left(\mu_{31}+\mu_{22}\right)$ and $\alpha_{1}=2\left(\mu_{4}-\mu_{22}\right)$. This motivates the following result.

Lemma 6.3. Let $\bar{\tau}$ be an exchangeable design on the simplex $\mathcal{T}$, with fourth order moments $\mu_{4}, \mu_{31}, \mu_{22}$. Then the weighted centroid design $\eta=\alpha_{1} \eta_{1}+\alpha_{2} \eta_{2}$, with weights $\alpha_{1}=2\left(\mu_{4}-\mu_{22}\right)$ and $\alpha_{2}=8\left(\mu_{31}+\mu_{22}\right)$, satisfies

$$
M(\eta) \geq M(\bar{\tau})
$$

with equality if and only if $\bar{\tau}=\eta$. 
Proof. The simplex restriction entails $2 \mu_{4}+8 \mu_{31}+6 \mu_{22}=1$ and $\alpha_{1}+\alpha_{2}=1$. Clearly we have $\alpha_{2} \geq 0$. We also have $\alpha_{1} \geq 0$, since the function $\psi\left(t_{1}, t_{2}\right)=\left(t_{1}^{2}-t_{2}^{2}\right)^{2}$ is nonnegative and satisfies $\alpha_{1}=\int \psi\left(t_{1}, t_{2}\right) d \bar{\tau} \geq 0$. Hence the weighted centroid design $\eta$ is well-defined.

It is easily seen that $\mu_{(2)}(\eta)=\mu_{(2)}(\bar{\tau})$. Using $\gamma=\frac{1}{2}\left(\mu_{31}-\mu_{22}\right) \geq 0$ we find $\mu_{4}(\eta)-\mu_{4}=$ $\gamma$. Lemma 6.1 establishes $M(\eta) \geq M(\bar{\tau})$. More precisely, we obtain $M(\eta)-M(\bar{\tau})=\gamma c c^{\prime} \geq$ 0 , with the same vector $c$ as in the proof of Lemma 6.1.

Equality holds if and only if $\gamma=0$. By Lemma 6.2, $\bar{\tau}$ then is a weighted centroid design. Since it shares the same moments with $\eta$, the two designs must be the same.

As an illustration of Lemma 6.3, we consider a family of exchangeable designs that depends on a single support parameter $r$,

$$
\tau_{r}\left(\begin{array}{c}
1-r \\
r
\end{array}\right)=\tau_{r}\left(\begin{array}{c}
r \\
1-r
\end{array}\right)=\frac{1}{2} \quad \text { for all } r \in\left[0, \frac{1}{2}\right] .
$$

Of course, for the limiting value $r=\frac{1}{2}$ we set $\tau_{r}\left(\frac{1}{2}, \frac{1}{2}\right)=1$. The weighted centroid design improving upon $\tau_{r}$ has weights that are quadratic in $r$,

$$
\alpha_{1}=(1-2 r)^{2}, \quad \alpha_{2}=4 r(1-r)
$$

The vertex points design, $\eta_{1}$, has $r=0$ and $\alpha_{1}=1$. The overall centroid design, $\eta_{2}$, has $r=\frac{1}{2}$ and $\alpha_{2}=1$, as it should. For $r \in\left(0, \frac{1}{2}\right)$ the weights $\alpha_{1}$ and $\alpha_{2}$ lie strictly between zero and one, and $M\left(\alpha_{1} \eta_{1}+\alpha_{2} \eta_{2}\right) \gtreqless M\left(\tau_{r}\right)$. The two elementary centroid designs $\eta_{1}$ and $\eta_{2}$ occur with equal weight $\frac{1}{2}$ at about $r \approx 0.15$.

The following theorem joins the partial steps together to obtain our main result on the Kiefer ordering, that the mixtures of the vertex points design $\eta_{1}$ and of the overall centroid design $\eta_{2}$ form a minimal complete class. Since the Kiefer ordering does not depend on the basis that is chosen for the regression function, we do not have to distinguish whether we refer to the K-model, or to the S-model.

Theorem 6.4. In the two-ingredient second-degree model, the set of weighted centroid designs

$$
\mathcal{C}=\left\{\alpha_{1} \eta_{1}+\alpha_{2} \eta_{2}:\left(\alpha_{1}, \alpha_{2}\right)^{\prime} \in \mathcal{T}\right\}
$$

constitutes a minimal complete class of designs for the Kiefer ordering.

Proof. Completeness of $\mathcal{C}$ means that for every design $\tau$ not in $\mathcal{C}$ there is a member $\eta$ in $\mathcal{C}$ that is Kiefer better than $\tau$. That is, we must show that $\eta$ is more informative than $\tau$, 
$M(\eta) \gg M(\tau)$, but that the two are not Kiefer equivalent, $M(\tau) \ngtr M(\eta)$. From Section 5 and with the weights from Lemma 6.3, the weighted centroid design $\eta=\alpha_{1} \eta_{1}+\alpha_{2} \eta_{2}$ satisfies $M(\eta) \geq M(\bar{\tau}) \prec M(\tau)$, that is, $M(\eta) \gg M(\tau)$.

If, in addition, we assume the reverse relation to hold true, $M(\tau) \gg M(\eta)$, then the two moment matrices are Kiefer equivalent and hence coincide (Pukelsheim 1993, page 356). This forces the two designs to be equal, contradicting the assumption that $\tau$ does not lie in $\mathcal{C}$. Therefore $\eta$ and $\tau$ are not Kiefer equivalent.

Minimal completeness of $\mathcal{C}$ means that no proper subset of $\mathcal{C}$ is complete. It suffices to show that, if we take away a member $\tau=\beta_{1} \eta_{1}+\beta_{2} \eta_{2}$ from $\mathcal{C}$, the remaining subclass is no longer complete. The proof is by contradiction. We assume that some design $\eta=$ $\alpha_{1} \eta_{1}+\alpha_{2} \eta_{2} \in \mathcal{C}$ does improve upon $\tau, M(\eta) \geq M(\tau)$. Lemma 6.1 says that $\eta$ and $\tau$ share the same lower order moments. The latter determine the weights uniquely, contradicting the assumption that the designs are distinct. Therefore the assumption $M(\eta) \geq M(\tau)$ cannot hold true, rendering the class $\mathcal{C}$ minimal complete.

The implication of the above is that any design which does not consist of a mixture of elementary centroid designs can be improved upon, in terms of symmetry and Loewner ordering, by using an appropriate combination of elementary centroid designs. Within the class of weighted centroid designs, however, other criteria would be needed to attain further improvement, for example, the determinant criterion.

\section{Three factors}

In the three-ingredient second-degree model, an exchangeable design $\bar{\tau}$ picks up an additional moment of order four, $\mu_{211}=\int t_{1}^{2} t_{2} t_{3} d \bar{\tau}$. Its K-moment matrix is

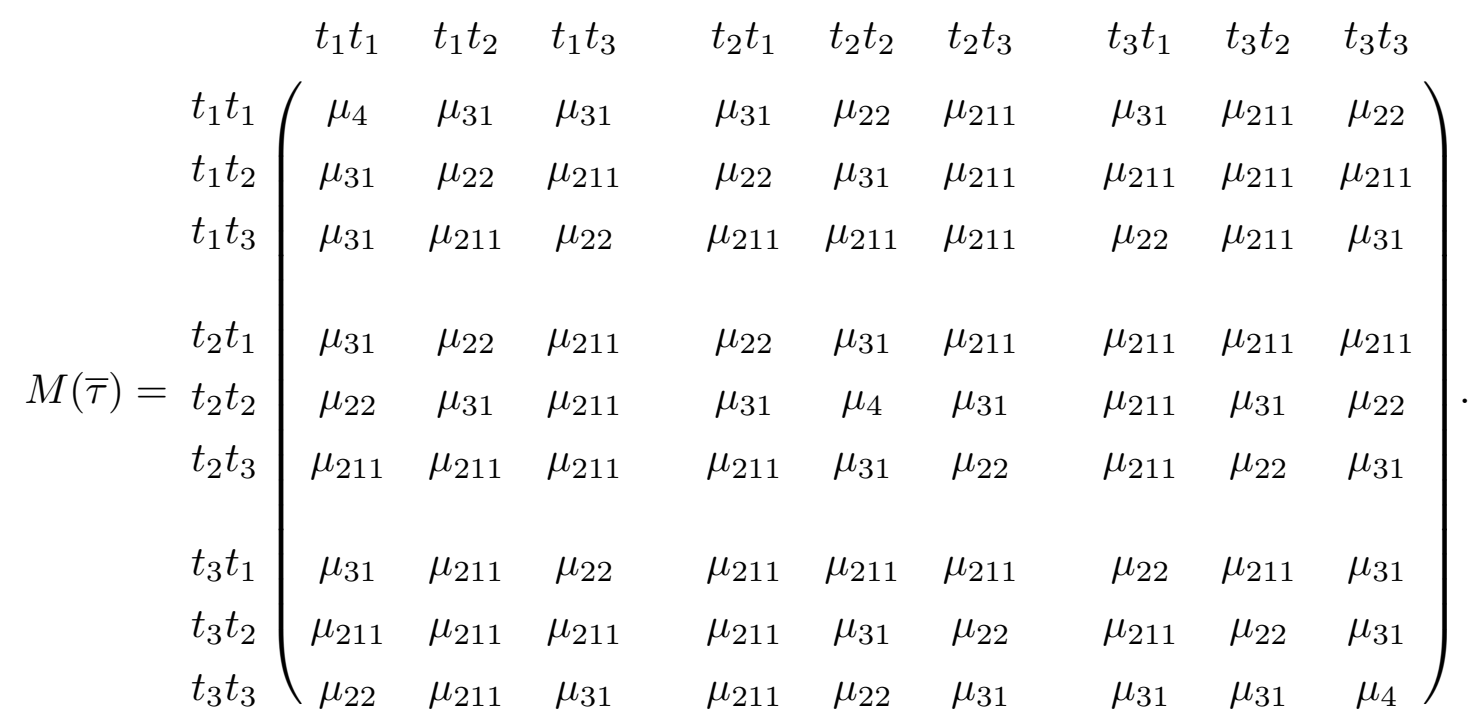


The Kronecker representation evidently causes three nullvectors, e.g.,

$$
(0,1,0,-1,0,0,0,0,0)^{\prime}, \quad(0,0,1,0,0,0,-1,0,0)^{\prime}, \quad(0,0,0,0,0,1,0,-1,0)^{\prime}
$$

The lower order moments are expressed through the fourth order moments as follows:

$$
\begin{aligned}
& \mu_{3}=\mu_{4}+2 \mu_{31}, \\
& \mu_{21}=\quad \mu_{31}+\mu_{22}+\mu_{211}, \\
& \mu_{111}= \\
&=\mu_{4}+4 \mu_{31}+2 \mu_{22}+2 \mu_{211} ; \\
& \mu_{2}=\mu_{3}+2 \mu_{21} \quad 2 \mu_{31}+2 \mu_{22}+5 \mu_{211} .
\end{aligned}
$$

The simplex restriction entails

$$
\begin{aligned}
& 1=3 \mu_{4}+24 \mu_{31}+18 \mu_{22}+36 \mu_{211}, \\
& 1=3 \mu_{3}+18 \mu_{21}+6 \mu_{111}, \\
& 1=3 \mu_{2}+6 \mu_{11} .
\end{aligned}
$$

The analogue of Lemma 6.1 uses the initial section of moments up to order three,

$$
\mu_{(3)}=\left(\mu_{2}, \mu_{11} ; \mu_{3}, \mu_{21}, \mu_{111}\right)^{\prime} .
$$

Lemma 7.1. Let $\eta$ and $\bar{\tau}$ be two exchangeable designs on the simplex $\mathcal{T}$. Then we have

$$
M(\eta) \geq M(\bar{\tau}) \quad \Longleftrightarrow \quad \mu_{(3)}(\eta)=\mu_{(3)}(\bar{\tau}), \quad \mu_{4}(\eta) \geq \mu_{4}(\bar{\tau}) .
$$

Proof. For the direct part we refine the argument in the proof of Lemma 6.1. Assume that $\Delta=M(\eta)-M(\bar{\tau})$ is nonnegative definite. Then $\left(1_{3} \otimes 1_{3}\right)^{\prime} \Delta\left(1_{3} \otimes 1_{3}\right)=0$ forces $\Delta\left(1_{3} \otimes 1_{3}\right)=0$ and equality of second order moments. Now we get

$$
\left(e_{1} \otimes 1_{3}\right)^{\prime} M(\eta)\left(e_{1} \otimes 1_{3}\right)=\int_{\mathcal{T}} t_{1}^{2} d \eta=\mu_{2}=\int_{\mathcal{T}} t_{1}^{2} d \bar{\tau}=\left(e_{1} \otimes 1_{3}\right)^{\prime} M(\bar{\tau})\left(e_{1} \otimes 1_{3}\right)
$$

This yields $\Delta\left(e_{1} \otimes 1_{3}\right)=0$, that is, $\int(t \otimes t) t_{1} d \eta=\int(t \otimes t) t_{1} d \bar{\tau}$. Hence the third order moments of $\eta$ and $\bar{\tau}$ are equal as well. This means $\mu_{(3)}(\eta)=\mu_{(3)}(\bar{\tau})$. In addition, we have $0 \leq\left(e_{1} \otimes e_{1}\right)^{\prime} \Delta\left(e_{1} \otimes e_{1}\right)=\mu_{4}(\eta)-\mu_{4}(\bar{\tau})$. 
For the converse part, equality of the third order moments forces the differences of the fourth order moments to even out according to, using $\gamma=\mu_{4}(\eta)-\mu_{4}(\bar{\tau}) \geq 0$,

$$
\begin{aligned}
\mu_{31}(\eta)-\mu_{31}(\bar{\tau}) & =-\frac{\gamma}{2} \\
\mu_{211}(\eta)-\mu_{211}(\bar{\tau}) & =0 \\
\mu_{22}(\eta)-\mu_{22}(\bar{\tau}) & =\frac{\gamma}{2}
\end{aligned}
$$

In terms of matrices this means

$$
M(\eta)-M(\bar{\tau})=\frac{\gamma}{2}\left(\begin{array}{ccccccccc}
2 & -1 & -1 & -1 & 1 & 0 & -1 & 0 & 1 \\
-1 & 1 & 0 & 1 & -1 & 0 & 0 & 0 & 0 \\
-1 & 0 & 1 & 0 & 0 & 0 & 1 & 0 & -1 \\
-1 & 1 & 0 & 1 & -1 & 0 & 0 & 0 & 0 \\
1 & -1 & 0 & -1 & 2 & -1 & 0 & -1 & 1 \\
0 & 0 & 0 & 0 & -1 & 1 & 0 & 1 & -1 \\
-1 & 0 & 1 & 0 & 0 & 0 & 1 & 0 & -1 \\
0 & 0 & 0 & 0 & -1 & 1 & 0 & 1 & -1 \\
1 & 0 & -1 & 0 & 1 & -1 & -1 & -1 & 2
\end{array}\right)=\frac{\gamma}{2} E \geq 0
$$

where $E=w_{12} w_{12}^{\prime}+w_{13} w_{13}^{\prime}+w_{23} w_{23}^{\prime}$ and $w_{i j}=\left(e_{i}-e_{j}\right) \otimes\left(e_{i}-e_{j}\right)=w_{j i}$.

The same results can be derived using the S-models. A three-ingredient second-degree S-moment matrix has the form

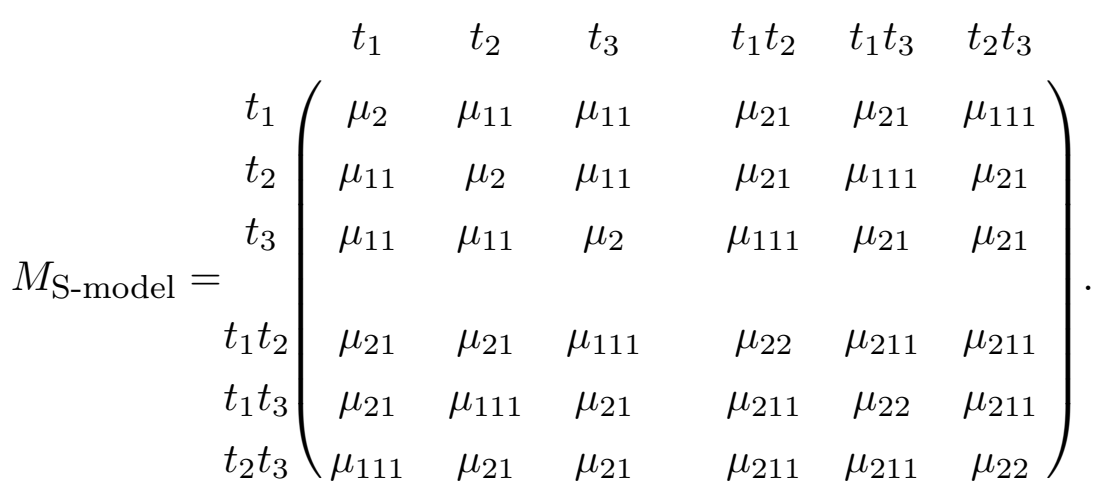

The difference between the S-moment matrices of the weighted centroid design $\eta$ and a 
given design $\bar{\tau}$ is

$$
\frac{\gamma}{2}\left(\begin{array}{llllll}
0 & 0 & 0 & 0 & 0 & 0 \\
0 & 0 & 0 & 0 & 0 & 0 \\
0 & 0 & 0 & 0 & 0 & 0 \\
0 & 0 & 0 & 1 & 0 & 0 \\
0 & 0 & 0 & 0 & 1 & 0 \\
0 & 0 & 0 & 0 & 0 & 1
\end{array}\right) \geq 0 .
$$

There are three elementary centroid designs: $\eta_{1}$ is supported on the vertices, $\eta_{2}$ on the edge midpoints, and $\eta_{3}$ on the overall centroid point,

$\eta_{1}\left(\begin{array}{l}1 \\ 0 \\ 0\end{array}\right)=\eta_{1}\left(\begin{array}{l}0 \\ 1 \\ 0\end{array}\right)=\eta_{1}\left(\begin{array}{c}0 \\ 0 \\ 1\end{array}\right)=\frac{1}{3}, \quad \eta_{2}\left(\begin{array}{c}\frac{1}{2} \\ \frac{1}{2} \\ 0\end{array}\right)=\eta_{2}\left(\begin{array}{c}\frac{1}{2} \\ 0 \\ \frac{1}{2}\end{array}\right)=\eta_{2}\left(\begin{array}{c}0 \\ \frac{1}{2} \\ \frac{1}{2}\end{array}\right)=\frac{1}{3}, \quad \eta_{3}\left(\begin{array}{c}\frac{1}{3} \\ \frac{1}{3} \\ \frac{1}{3}\end{array}\right)=1$.

The moments of order four of these designs are, respectively:

$$
\begin{aligned}
& \mu_{4}\left(\eta_{1}\right)=\frac{1}{3}, \\
& \mu_{4}\left(\eta_{2}\right)=\frac{1}{24}, \\
& \mu_{4}\left(\eta_{3}\right)=\frac{1}{81}, \\
& \mu_{31}\left(\eta_{1}\right)=\mu_{22}\left(\eta_{1}\right)=0 \text {, } \\
& \mu_{31}\left(\eta_{2}\right)=\mu_{22}\left(\eta_{2}\right)=\frac{1}{48}, \\
& \mu_{31}\left(\eta_{3}\right)=\mu_{22}\left(\eta_{3}\right)=\frac{1}{81}, \\
& \mu_{211}\left(\eta_{1}\right)=0, \\
& \mu_{211}\left(\eta_{2}\right)=0, \\
& \mu_{211}\left(\eta_{3}\right)=\frac{1}{81} .
\end{aligned}
$$

Definition. For weights $\alpha_{1}, \alpha_{2}, \alpha_{3} \geq 0$ with $\alpha_{1}+\alpha_{2}+\alpha_{3}=1$, the design $\eta=\alpha_{1} \eta_{1}+$ $\alpha_{2} \eta_{2}+\alpha_{3} \eta_{3}$ is called a weighted centroid design.

The following Lemma 7.2 is a close analogue to Lemma 6.2.

Lemma 7.2. Let $\bar{\tau}$ be an exchangeable design on the simplex $\mathcal{T}$. Then we have

$$
\mu_{31}(\bar{\tau}) \geq \mu_{22}(\bar{\tau})
$$

with equality if and only if $\bar{\tau}$ is a weighted centroid design.

Proof. On the simplex $\mathcal{T}$, the symmetric function

$$
\psi\left(t_{1}, t_{2}, t_{3}\right)=t_{1} t_{2}\left(t_{1}-t_{2}\right)^{2}+t_{1} t_{3}\left(t_{1}-t_{3}\right)^{2}+t_{2} t_{3}\left(t_{2}-t_{3}\right)^{2}
$$

is nonnegative, giving $\mu_{31}(\bar{\tau})-\mu_{22}(\bar{\tau})=\frac{1}{6} \int \psi\left(t_{1}, t_{2}, t_{3}\right) d \bar{\tau} \geq 0$.

Equality holds if and only if $\psi$ vanishes for all support points of $\bar{\tau}$. Hence the support points of $\bar{\tau}$ must be vertices, edge midpoints, or the overall centroid point. Because of exchangeability, $\bar{\tau}$ is a weighted centroid design. 
For the case when $\eta=\alpha_{1} \eta_{1}+\alpha_{2} \eta_{2}+\alpha_{3} \eta_{3}$ is a weighted centroid design, we can now express the difference between the pure fourth order moments of $\eta$ and $\bar{\tau}$ in the converse part of the proof of Lemma 7.1 solely in terms of moments of $\bar{\tau}$,

$$
\gamma=\mu_{31}(\bar{\tau})-\mu_{22}(\bar{\tau}) \geq 0
$$

In order to find an appropriate set of weights we equate selected moments of order lower than four:

$$
\begin{array}{rlrl}
\mu_{1}(\eta) & \equiv \frac{1}{3} \alpha_{1}+\frac{1}{3} \alpha_{2}+\frac{1}{3} \alpha_{3} & =\frac{1}{3} \\
\mu_{11}(\eta) & \equiv & \frac{1}{12} \alpha_{2}+\frac{1}{9} \alpha_{3} & =\mu_{11}, \\
\mu_{111}(\eta) & \equiv & \frac{1}{27} \alpha_{3} & =\mu_{111} .
\end{array}
$$

The solutions are $\alpha_{3}=27 \mu_{111}, \alpha_{2}=12\left(\mu_{11}-3 \mu_{111}\right), \alpha_{1}=1-\alpha_{2}-\alpha_{3}$. When the lower order moments are expressed using fourth order moments, these weights are seen to be the ones given in the following lemma.

Lemma 7.3. Let $\bar{\tau}$ be an exchangeable design on the simplex $\mathcal{T}$, with fourth order moments $\mu_{4}, \mu_{31}, \mu_{22}, \mu_{211}$. Then the weighted centroid design $\eta=\alpha_{1} \eta_{1}+\alpha_{2} \eta_{2}+\alpha_{3} \eta_{3}$, with weights $\alpha_{1}=3\left(\mu_{4}-2 \mu_{22}+\mu_{211}\right), \alpha_{2}=24\left(\mu_{31}+\mu_{22}-2 \mu_{211}\right), \alpha_{3}=81 \mu_{211}$, satisfies

$$
M(\eta) \geq M(\bar{\tau})
$$

with equality if and only if $\bar{\tau}=\eta$.

Proof. The relation $3 \mu_{4}+24 \mu_{31}+18 \mu_{22}+36 \mu_{211}=1$ entails $\alpha_{1}+\alpha_{2}+\alpha_{3}=1$. Clearly we have $\alpha_{3} \geq 0$. We also have $\alpha_{2} \geq 0$, since the function $\phi\left(t_{1}, t_{2}, t_{3}\right)=12\left(t_{1}-t_{2}\right)^{2}\left(t_{1} t_{2}+2 t_{3}^{2}\right)$ is nonnegative and integrates to $\alpha_{2}$. For $\alpha_{1}$, we use the symmetric function

$$
\psi\left(t_{1}, t_{2}, t_{3}\right)=t_{1}^{4}+t_{2}^{4}+t_{3}^{4}-2 t_{1}^{2} t_{2}^{2}-2 t_{1}^{2} t_{3}^{2}-2 t_{2}^{2} t_{3}^{2}+t_{1}^{2} t_{2} t_{3}+t_{1} t_{2}^{2} t_{3}+t_{1} t_{2} t_{3}^{2} .
$$

It can be shown that, on the simplex, $\psi$ is nonnegative. This ensures $\alpha_{1} \geq 0$. Hence the weighted centroid design $\eta$ is well-defined.

We readily verify $\mu_{(3)}(\eta)=\mu_{(3)}(\bar{\tau})$. Using $\gamma=\mu_{31}-\mu_{22} \geq 0$ we find $\mu_{4}(\eta)-\mu_{4}=\gamma$. Lemma 7.1 establishes $M(\eta) \geq M(\bar{\tau})$. More precisely, we obtain $M(\eta)-M(\bar{\tau})=\frac{\gamma}{2} E \geq 0$, with the same matrix $E$ as in the proof of Lemma 7.1.

Equality holds if and only if $\gamma=0$. By Lemma 7.2, $\bar{\tau}$ then is a weighted centroid design. Since it shares the same moments with $\eta$, the two design must be the same. 
As an illustration of Lemma 7.3 we consider the one-parameter family of exchangeable designs that is given by

$$
\tau_{r}\left(\begin{array}{c}
1-2 r \\
r \\
r
\end{array}\right)=\tau_{r}\left(\begin{array}{c}
r \\
1-2 r \\
r
\end{array}\right)=\tau_{r}\left(\begin{array}{c}
r \\
r \\
1-2 r
\end{array}\right)=\frac{1}{3} \quad \text { for all } r \in\left[0, \frac{1}{2}\right] .
$$

This family includes the vertex points design, $\tau_{0}=\eta_{1}$, and the edge midpoints design, $\tau_{1 / 2}=\eta_{2}$. Of course, for the value $r=\frac{1}{3}$ we take $\tau_{r}$ to be the overall centroid design $\eta_{3}$. The weighted centroid design $\eta$ from Lemma 7.3 then has weights that are cubic in $r$,

$$
\alpha_{1}(r)=(1-2 r)(1-3 r)^{2}, \quad \alpha_{2}(r)=8 r(1-3 r)^{2}, \quad \alpha_{3}(r)=27 r^{2}(1-2 r) .
$$

The vertex points design has $\alpha_{1}(0)=1$, the edge midpoints design has $\alpha_{2}\left(\frac{1}{2}\right)=1$, and the overall centroid design has $\alpha_{3}\left(\frac{1}{3}\right)=1$, as one would expect.

As another example, we consider for the initial design the $\{3,3\}$ simplex lattice design (Scheffé 1958; Cornell 1990, page 23) assigning weight 1/10 to each of the 10 points

$$
\left(\begin{array}{l}
1 \\
0 \\
0
\end{array}\right),\left(\begin{array}{l}
0 \\
1 \\
0
\end{array}\right),\left(\begin{array}{l}
0 \\
0 \\
1
\end{array}\right),\left(\begin{array}{c}
\frac{2}{3} \\
\frac{1}{3} \\
0
\end{array}\right),\left(\begin{array}{c}
\frac{2}{3} \\
0 \\
\frac{1}{3}
\end{array}\right),\left(\begin{array}{c}
\frac{1}{3} \\
\frac{2}{3} \\
0
\end{array}\right),\left(\begin{array}{c}
\frac{1}{3} \\
0 \\
\frac{2}{3}
\end{array}\right),\left(\begin{array}{c}
0 \\
\frac{1}{3} \\
\frac{2}{3}
\end{array}\right),\left(\begin{array}{c}
0 \\
\frac{2}{3} \\
\frac{1}{3}
\end{array}\right),\left(\begin{array}{c}
\frac{1}{3} \\
\frac{1}{3} \\
\frac{1}{3}
\end{array}\right) .
$$

This initial design has moments $\mu_{4}=\frac{116}{810}, \mu_{31}=\frac{11}{810}, \mu_{22}=\frac{9}{810}, \mu_{211}=\frac{1}{810}$. The sixth root of the determinant of the corresponding S-moment matrix is equal to 0.0352 .

A better design, from the Loewner ordering point of view, is the weighted centroid design $\eta$, with weights $\alpha_{1}=\frac{11}{30}, \alpha_{2}=\frac{16}{30}, \alpha_{3}=\frac{3}{30}$. The scalar $\frac{\gamma}{2}$ that determines the moment matrix improvement equals $1 / 810$. The moments are $\mu_{4}=\frac{118}{810}, \mu_{31}=\mu_{22}=$ $\frac{10}{810}, \mu_{211}=\frac{1}{810}$. The S-moment matrix of this design has sixth root of the determinant equal to 0.0388 . Thus it is 10 percent more D-efficient than the initial design. The improved design uses only seven support points, namely, the three vertices, the three edge midpoints, and the overall centroid point. However, the weights $\alpha_{1}, \alpha_{2}, \alpha_{3}$ are such that the design is not realizable for sample size $n=10$.

As a remedy, we consider a slight shift of weights to $\beta_{1}=\frac{3}{10}, \beta_{2}=\frac{6}{10}, \beta_{3}=\frac{1}{10}$. This suggests reallocating the 10 observations that the simplex lattice design calls for, and to take one observation of each single-component mixture, two observations of each binary blend of equal proportion, and one observation with all three ingredients at equal proportions. This design has moments $\mu_{4}=\frac{102.25}{810}, \mu_{31}=\mu_{22}=\frac{11.125}{810}, \mu_{211}=\frac{1}{810}$. The 
sixth root of the determinant of its S-moment matrix is 0.0371. So this compromise design is 5 percent more D-efficient than the initial design.

The above efficiency comparisons are made with respect to a second order model. In fact, the $\{3,3\}$ simplex lattice design could be used to fit a cubic regression function and, it could be argued, provides lack of fit degrees of freedom, if only a quadratic model is fitted. These considerations are of course important in some contexts.

Again we conclude that, in the Kiefer design ordering, it suffices to restrict attention to the convex combinations of the vertex points design $\eta_{1}$, the edge midpoints design $\eta_{2}$, and the overall centroid design $\eta_{3}$. Since the representation of the second-degree regression function is immaterial, we do not need to distinguish between the K-model and the S-model.

Theorem 7.4. In the three-ingredient second-degree model, the set of weighted centroid designs

$$
\mathcal{C}=\left\{\alpha_{1} \eta_{1}+\alpha_{2} \eta_{2}+\alpha_{3} \eta_{3}:\left(\alpha_{1}, \alpha_{2}, \alpha_{3}\right)^{\prime} \in \mathcal{T}\right\}
$$

constitutes a minimal complete class of designs for the Kiefer ordering.

Proof. The completeness part is established just as in Theorem 6.4. For minimal completeness, we remove a weighted centroid design $\tau$ from $\mathcal{C}$ and assume that $\eta \in \mathcal{C}$ improves upon $\tau, M(\eta) \geq M(\tau)$. By Lemma 7.1 the two designs share the same lower order moments. The latter determine the weights uniquely, contradicting the assumption that $\tau$ and $\eta$ are distinct. Hence the class $\mathcal{C}$ is minimal complete.

Cases $m \geq 4$ are considerably more complicated and are discussed in Draper, Heiligers and Pukelsheim (1998).

\section{Brief review of related literature}

Atwood (1969) deduces (page 1574) from his Theorem 2.2 that any $D$-optimal design is supported on the centroid points of the faces of the simplex (i.e., on the barycenters). The second-degree $D$-optimal design uses the elementary centroid designs $\eta_{1}$ and $\eta_{2}$, and the third-degree $D$-optimal design uses $\eta_{1}, \eta_{2}$, and $\eta_{3}$. He then shows (page 1575) that the fourth-degree $D$-optimal design needs support points other than those provided by $\eta_{1}$, $\eta_{2}, \eta_{3}$, and $\eta_{4}$. In fact, his arguments cover any degree greater than three, in models for which the number of factors exceeds the degree, $3<d<m$. 
Chan (1995) helpfully summarizes 104 references which deal with optimal design for mixture models. Section 1 discusses the variety of models used, Section 2 presents optimality criteria, and Section 3 reviews specific optimal designs that have been obtained for various circumstances.

Chan, Guan and Zhang (1998) obtain A-optimal designs for a mixture model due to Darroch and Waller (1985) that contains $2 m$ terms involving $t_{i}$ and $t_{i}^{2}$. The designs combine $m$ equally weighted points that are centers of faces with other design points that vary with $m$.

Chan, Meng, Jiang and Guan (1998) investigate quadratic models with $2 m$ terms in $t_{i}$ and $t_{i}^{2}$, and cubic models with $3 m$ terms in $t_{i}, t_{i}^{2}$ and $t_{i}^{3}(i=1, \ldots, m)$ in terms of axial designs. These designs consist of two symmetric axial point sets for the quadratic model, and three such sets for the cubic model. D-optimality is achieved by taking one set as the corner points, with specified levels for the other point set(s).

Chan and Sandhu (1999): For $m=3$ components, the $A-, D$ - and $E$-optimality properties of an orthogonally blocked eight point design of P.W.M. John are considered.

Cheng (1995) derives results for the Kiefer ordering when, for the $m \times m$ moment matrices, the induced group is the full permutation group, $\mathcal{Q}=\operatorname{Perm}(m)$. Hence our discussion of the first-degree model is subsumed by his results. For second- and higher degree models, however, a proper subgroup of the permutation group is induced, $\mathcal{Q} \neq$ Perm $(m)$, whence our results cannot be derived from his.

Cornell (1990) is the definitive text on Scheffé-type mixture models.

Cornell and Ramsey (1998) construct a mixture model with the aim of representing in every mixture one or more minor components, selected from a category of major components. The inter-category and between-category blending properties are modeled by forming the Kronecker product of appropriate submodels.

Cox (1971) provides an alternative to the S-models, by using a polynomial representation that admits the interpretation of standard operating conditions as a base.

Crosier (1984) uses a transformation (page 211) to reduce the ill-conditioning in fitting Scheffé's polynomials. The paper may be taken as an indication to study other transformations of the Scheffé model, thus encouraging our K-model approach.

Darroch and Waller (1985): For $m=3$ mixture ingredients, various models are discussed, including models whose forms involve the addition of submodels containing a single 
ingredient. Ways of representing interaction effects by "non-blandness" parameters are then investigated.

Dean, Lewis, Prescott and Draper (1992): In Draper, Prescott, Lewis, Dean, John, and Tuck (1993), the symbolic algebra system program, MAPLE, can be used to obtain D-optimal designs. The brute force of the computer program is often too complicated and causes failure. Neater and more thoughtful programming is essential. This paper discusses some of the problems and how they were solved.

Dette (1997) observes that, in polynomial regression, the entries of the moment matrix may turn out to be scaled quite differently. As a remedy, he proposes matching the distinct scaling by choice of an appropriately "standardized" optimality criterion. We address the same intrinsic problem but instead suggest a transition from S- to K-models.

Draper and Pukelsheim (1998a) provide a synopsis of the Kiefer ordering of rotatable designs on the ball, for first-, second-, and third-degree models, and demonstrate the advantages of Kronecker algebra.

Draper and Pukelsheim (1998b) point out inhomogeneity of the entries of the moment matrix in S-models, and propose, as an alternative, a switch to the K-models whose moment matrices are homogeneous. The paper shows how the S- and K-parameters transform.

Draper, Prescott, Lewis, Dean, John and Tuck (1993): Latin squares of side four are used to construct mixture designs in orthogonal blocks. Selected squares are each paired with their "mate", namely with a square with which it can satisfy the block orthogonality condition. A design is built up by using two or more pairs of mates and adding an additional run, usually $(0.25,0.25,0.25,0.25)$ to each block. A versatile selection of designs can thus be found. D-optimal designs, with points on the edges, can be selected. An industrial example illustrates the practical uses of such designs.

Farrell, Kiefer and Walbran (1967): For the third-degree model with three factors, the $D$-optimal design is shown (page 119) to assign equal mass to the ten points consisting of the three vertices, the simplex centroid point $(1 / 3,1 / 3,1 / 3)^{\prime}$, and the six permutations of $(r, 1-r, 0)^{\prime}$ with $r=(1+1 / \sqrt{5}) / 2$. Verification of the equivalence theorem is based on the orthogonalization of the regression functions relative to the optimal design.

Gaffke and Heiligers (1996) have a comprehensive review of invariance in design problems. Lemma 3.3 (page 1167) was initially instrumental to our Lemmas 6.1 and 7.1, but has been superseded by direct arguments.

Galil and Kiefer (1979) is a seminal paper for optimality of designs in second-degree 
S-model, with extensive results when the criterion is one of the matrix means. Optimal designs must be supported by the centroids of the faces of the simplex, and must be invariant under permutations of the coordinates of their support points (page 448). Whereas we prefer to rely on the moments $\mu_{11}, \mu_{111}, \mu_{1111}$, they pick (page 448) $\mu_{11}, \mu_{21}, \mu_{22}$. The geometry of the polyhedron that is generated by the moment vectors $\left(\mu_{11}, \mu_{21}, \mu_{22}\right)^{\prime}$ is discussed (page 451).

Gorman and Hinman (1962) provide an early illustration of the applicability of mixture models as introduced by Scheffé (1958) with formulas up to fourth-degree.

Hilgers and Bauer (1995) extend the simplex towards the origin, which allows the modelling of a total amount in addition to the mixtures of the ingredients. The model then includes an overall mean $\theta_{0}$. For higher degree models, the authors aim at generalizing the results of Atwood (1969).

Kiefer (1959) discusses admissibility of designs, and essentially and minimal complete classes of designs relative to the Loewner matrix ordering (page 286).

Kiefer (1961): For the general $m$-ingredients second-degree S-model, the $D$-optimal design is shown (page 320) to assign equal weight to the vertices and the centroids of the edges. The method of proof is to determine a system of polynomials that are orthonormal under the optimal design, and then verify the Equivalence Theorem. For the special case of the three-ingredients second-degree model, the mixture $\alpha \eta_{1}+(1-\alpha) \eta_{2}$ with $\alpha=(9-\sqrt{17}) / 8$ is shown (page 322) to be $D$-optimal for the subsystem of parameters that belongs to the quadratic terms in the S-model. For the three-ingredients third-degree model, various partial results are discussed.

Kiefer (1975a) introduces the notion of universal optimality. See also Pukelsheim (1993).

Kiefer (1975b) is about invariance reduction in the second-degree S-model. As a consequence of the Equivalence Theorem for the matrix mean criteria, Kiefer concludes that any optimal designs can have only the centroids of the faces for its support points. He therefore restricts the search for optimal designs to convex combinations of elementary centroid designs.

Kiefer (1978): Asymptotics for $m$-ingredients second-degree S-models, as the number of ingredients tends to infinity, $m \rightarrow \infty$, are discussed. For the smallest-eigenvalue criterion, formulas for the limiting weights $\alpha_{j}$ of the elementary centroid designs $\eta_{j}$ are characterized (page 1356). Kiefer then concentrates on the basic solutions in which only 
four of the weights are positive. It emerges that $\alpha_{1}$ and $\alpha_{2}$ must always be positive, the remaining two weights then being $\alpha_{j}$ and $\alpha_{h}$ with $2<j<h$. Formulas are given when $j=2$, or $j=3$ (page 1357).

Laake (1975) discusses optimality in the second-degree S-model, relative to the integrated variance criterion. In the class of simplex lattice design this leads to equal weights on the vertices, and equal weights on the edge midpoints. The total vertex weight is tabulated for some selected number of factors $m \geq 4$. Results for the third-degree model are quoted from his 1973 thesis at the University of Oslo. The same program is then carried out for the class of simplex centroid designs.

Lewis, Dean, Draper and Prescott (1994): Latin squares of general side $q$ are explored for their use in orthogonally blocked mixture designs. Some general results for any $q$ are established and some examples for 6 and 8 ingredients shown.

$\operatorname{Lim}(1990)$ is about $D$-optimal design in the $m$-ingredients third-degree model, elaborating on the approach for the $m=3$ result of Farrell, Kiefer and Walbran (1967). Case $m=4$ is solved conclusively, the cases $m=5, \ldots, 10$ are solved numerically.

Liu and Neudecker (1995) discuss optimality under the integrated variance criterion, for S-models of arbitrary degree. They use the notion of a "weighted simplex-centroid design" (page 254). In Liu and Neudecker (1997, page 55), weighted simplex-centroid designs are applied to Becker's models.

Mikaeili (1988): Optimality in third-degree S-models, under the integrated variance criterion, the determinant criterion, and the average-variance criterion is discussed. The class of competing designs is taken to depend on a single parameter $a$, in that the edge midpoints are replaced by the vectors $(a, 1-a, 0, \ldots, 0)^{\prime}$ and permutations thereof. The method is to find orthonormal polynomials under the optimal design.

Mikaeili (1989) finds the $D$-optimal design in a third-degree S-model with cubic threeingredients effects. It puts uniform weights on the vertices and the Farrell-Kiefer-Walbran (1967) edge support points $(a, 1-a, 0, \ldots, 0)^{\prime}$ with $a=(1+1 / \sqrt{5}) / 2$.

Mikaeili (1993) provides verification of the numerical evidence of Lim (1990). He uses identities between symmetric functions on the simplex, and also employs orthonormalization under the optimal design.

Prescott, Draper, Dean and Lewis (1993): Latin squares of side five are used to construct mixture designs in orthogonal blocks. This extends the work in Dean, Draper, 
John, Lewis, Prescott and Tuck (1993) in a parallel development (but excluding the optimal choices).

Prescott, Draper, Lewis and Dean (1997): D-optimal mixture designs for five factors, in orthogonal blocks, are obtained, thus developing the material in Dean, Draper, Lewis and Prescott (1993).

Pukelsheim (1993) defines (page 352) the Kiefer ordering as an extension of Kiefer's (1975) notion of "universal optimality". Examples include balanced incomplete block designs, multiple linear regression designs on the unit cube, and rotatable designs on the ball. See also Draper and Pukelsheim (1998a).

Scheffé (1958) is a seminal paper on mixture models and introduces the simplex lattice designs. Scheffé (1963) introduces the simplex centroid designs.

Smith and Beverly (1997) discuss the shortcomings of canonical polynomials, and instead use the polynomial representation of Cox (1971). Given the base point of the Cox polynomial, designs can be generated using a program supplied by the authors.

Uranisi (1964) extends a result of Kiefer (1961, page 323) from $m=2$ to $m \geq 3$. This says that the weighted centroid design assigning equal weights to the union of the support points of all elementary centroid designs is $D$-optimal, for the special cubic mixture model.

\section{Acknowledgements}

We would like gratefully to acknowledge partial support from the Alexander-vonHumboldt-Stiftung through a Max-Planck-Award for cooperative research. We thank the referees for several valuable suggestions.

\section{References}

Atwood, C.L. (1969). Optimal and efficient designs of experiments. Annals of Mathematical Statistics 40 1570-1602.

Chan, L.-Y. (1995). A review on optimal design for mixture models. In Five Decades as a Mathematician and Educator-On the 80th Birthday of Professor Yung-Chow Wong (K.-Y. Chan and M.-C. Liu, eds.) 45-88. World Scientific, Singapore.

Chan, L.-Y., Guan, Y.-N. and Zhang, C.-Q. (1998). A-optimal designs for an additive quadratic mixture model. Statistica Sinica 8 979-990.

Chan, L.Y., Meng, J.H., Jiang, Y.C. and Guan, Y.N. (1998). D-optimal axial designs for quadratic and cubic additive mixture models. Australian \& New Zealand Journal of Statistics 40 359-371.

Chan, L.Y. and Sandhu, M.K. (1999). Optimal orthogonal block designs for a quadratic mixture model for three components. Journal of Applied Statistics, in press. 
Cheng, C.-S. (1995). Complete class results for the moment matrices of designs over permutation-invariant sets. Annals of Statistics $\mathbf{2 3} 41-54$.

Cornell, J.A. (1990). Experiments with Mixtures. Designs, Models, and the Analysis of Mixture Data. Second Edition. Wiley, New York.

Cornell, J.A. and Ramsey, P.J. (1998). A generalized mixture model for categorized-components problems with an application to a photoresist-coating experiment. Technometrics 40 48-61.

Cox, D.R. (1971). A note on polynomial response functions for mixtures. Biometrika 58 55-159.

Crosier, R.B. (1984). Mixture experiments: Geometry and pseudocomponents. Technometrics 26 $209-216$.

Darroch, J.N. and Waller, J. (1985). Additivity and interaction in three-component experiments with mixtures. Biometrika $\mathbf{7 2}$ 153-163.

Dean, A.M., Lewis, S.M., Prescott, P. and Draper, N.R. (1992). Use of a symbolic algebra computer system to investigate the properties of mixture designs in orthogonal blocks. In Computational Statistics, Volume 2. Proceedings of the Tenth Symposium on Computational Statistics, Neuchâtel, Switzerland (Y. Dodge and J. Whittaker, eds.) 215-220. Physica, Heidelberg.

Dette, H. (1997). Designing experiments with respect to 'standardized' optimality criteria. Journal of the Royal Statistical Society Series B 59 97-110.

Draper, N.R., Heiligers, B. and Pukelsheim, F. (1998). Kiefer ordering of simplex designs for second-degree mixture models with four or more ingredients. Report 403, Institut für Mathematik, Universität Augsburg.

Draper, N.R., Prescott, P., Lewis, S.M., Dean, A.M., John, P.W.M. and Tuck, M.G. (1993). Mixture designs for four components in orthogonal blocks. Technometrics 35 268-276.

Draper, N.R. and Pukelsheim, F. (1998a). Polynomial representations for response surface modeling. New Developments and Applications in Experimental Design. (N. Flournoy, W.F. Rosenberger and W.K. Wong, eds.) Institute of Mathematical Statistics, Lecture Notes Series, forthcoming.

Draper, N.R. and Pukelsheim, F. (1998b). Mixture models based on homogeneous polynomials. Journal of Statistical Planning and Inference $\mathbf{7 1} 303-311$.

Farrell, R.H., Kiefer, J.C. and Walbran, A. (1967). Optimum multivariate designs. In Proceedings of the Fifth Berkeley Symposium on Mathematical Statistics and Probability, Berkeley, CA 1965 and 1966, Volume 1 (L.M. Le Cam and J. Neyman, eds.) 113-138. University of California, Berkeley CA. Also in: Kiefer (CP) 247-272.

Gaffke, N. and Heiligers, B. (1996). Approximate designs for polynomial regression: Invariance, admissibility, and optimality. In Handbook of Statistics, Volume 13 (S. Ghosh and C.R. Rao, eds.) 1149-1199. Elsevier, New York.

Galil, Z. and Kiefer, J. (1977). Comparison of simplex designs for quadratic mixture models. Technometrics 19 445-453. Also in: Kiefer (CP) 417-425.

Gorman, J.W. and Hinman, J.E. (1962). Simplex lattice designs for multicomponent system. Technometrics 4 463-487.

Hilgers, R.-D. and Bauer, P. (1995). Optimal designs for mixture amount experiments. Journal of Statistical Planning and Inference 48 241-246.

Kiefer, J.C. (1959). Optimum experimental designs. Journal of the Royal Statistical Society Series B $\mathbf{2 1}$ 272-304. Also in: Kiefer (CP) 54-101.

Kiefer, J.C. (1961). Optimum designs in regression problems, II. Annals of Mathematical Statistics 32 298-325. Also in: Kiefer (CP) 134-161. 
Kiefer, J.C. (1975a). Construction and optimality of generalized Youden designs. In A Survey of Statistical Design and Linear Models (J.N. Srivastava, ed.) 333-353. North-Holland, Amsterdam. Also in: Kiefer (CP) 345-353.

Kiefer, J.C. (1975b). Optimal design: Variation in structure and performance under change of criterion. Biometrika 62 277-288. Also in: Kiefer (CP) 367-378.

Kiefer, J.C. (1978). Asymptotic approach to families of design problems. Communications in StatisticsTheory and Methods A7 1347-1362. Also in: Kiefer (CP) 431-446.

Kiefer, J.C. (CP). Jack Carl Kiefer Collected Papers III. Design of Experiments. (L.D. Brown, I. Olkin, J. Sacks and H.P. Wynn, eds.). Springer, New York, 1985.

Laake, P. (1975). On the optimal allocation of observations in experiments with mixtures. Scandinavian Journal of Statistics 2 153-157.

Lewis, S.M., Dean, A.M., Draper, N.R. and Prescott, P. (1994). Mixture design for $q$ components in orthogonal blocks. Journal of the Royal Statistical Society Series B 56 457-467.

Lim, Y.B. (1990). D-optimal design for cubic polynomial regression on the $q$-simplex. Journal of Statistical Planning and Inference 25 141-152.

Liu, S. and Neudecker, H. (1995). A V-optimal design for Scheffé's polynomial model. Statistics and Probability Letters 23 253-258.

Liu, S. and Neudecker, H. (1997). Experiments with mixtures: Optimal allocations for Becker's models. Metrika 45 53-66.

Mikaeili, F. (1988). Allocation of measurements in experiments with mixtures. Keio Science and Technology Reports 41 25-37.

Mikaeili, F. (1989). D-optimum design for cubic without 3-way effect on the simplex. Journal of Statistical Planning and Inference 21 107-115.

Mikaeili, F. (1993). D-optimum design for full cubic on $q$-simplex. Journal of Statistical Planning and Inference 35 121-130.

Prescott, P., Draper, N.R., Dean, A.M. and Lewis, S.M. (1993). Mixture designs for five components in orthogonal blocks. Journal of Applied Statistics 20 105-117.

Prescott, P., Draper, N.R., Lewis, S.M. and Dean, A.M. (1997). Further properties of mixture designs for five components in orthogonal blocks. Journal of Applied Statistics 24 147-156.

Pukelsheim, F. (1993). Optimal Design of Experiments. Wiley, New York.

Scheffé, H. (1958). Experiments with mixtures. Journal of the Royal Statistical Society Series B $20344-$ 360.

Scheffé, H. (1963). The simplex-centroid design for experiments with mixtures. Journal of the Royal Statistical Society Series B 25 235-251.

Smith, W.F. and Beverly, T.A. (1997). Generating linear and quadratic Cox mixture models. Journal of Quality Technology 29 211-219.

Uranisi, H. (1964). Optimum design for the special cubic regression on the q-simplex. Mathematical Reports of College of General Education of Kyushu University 1 7-12. 